\title{
Electron molecule collisions calculations using the R-matrix method
}

\author{
Jonathan Tennyson* Jimena D. Gorfinkiel, I. Rozum, \\ Cynthia S. Trevisan and Natalia Vinci \\ Department of Physics and Astronomy, University College London, Gower Street, \\ London WC1E $6 B T, U K$
}

\begin{abstract}
The R-matrix method provides a complete theoretical framework for the treatment of low energy electron collisions. Recent results obtained with the UK R-matrix codes are presented focusing on electron impact electronic excitation of water and the $\mathrm{CF}$ radical, electron impact dissociation of molecular hydrogen and its isotopomers, and the dissociative recombination of the $\mathrm{CO}^{2+}$ dication. Examples of other processes studied in recent calculations are also given.
\end{abstract}

\section{Introduction}

Low-energy collisions with molecules can result in a variety of different processes. Defining low energies as ones in which the projectile electron has insufficient energy to ionize the target molecule $(A B)$, the following can occur:

Elastic Scattering

$\mathrm{AB}+e^{-} \rightarrow \mathrm{AB}+e^{-}$

Electronic Excitation

$\mathrm{AB}+e^{-} \rightarrow \mathrm{AB}^{*}+e^{-}$

Vibrational Excitation

$\mathrm{AB}\left(v^{\prime \prime}=0\right)+e^{-} \rightarrow \mathrm{AB}\left(v^{\prime}\right)+e^{-}$

Rotational Excitation

$\mathrm{AB}\left(N^{\prime \prime}\right)+e^{-} \rightarrow \mathrm{AB}\left(N^{\prime}\right)+e^{-}$

* Email: j.tennyson@ucl.ac.uk; Fax: (+44) 2076792564

Preprint submitted to Elsevier Science

28 January 2003 
Dissociative attachment / recombination

$$
\begin{aligned}
\mathrm{AB}+e^{-} \rightarrow & \mathrm{A}^{-}+\mathrm{B} \\
& \mathrm{A}+\mathrm{B}^{-}
\end{aligned}
$$

Impact dissociation

$$
\mathrm{AB}+e^{-} \rightarrow \mathrm{A}+\mathrm{B}+e^{-}
$$

A common feature of all these processes is that they can be considered to go via a common intermediary, $A B^{-}$. The R-matrix method for treating electronmolecule collisions is designed, described in this article, to obtain accurate wavefunctions for this intermediary and hence gives a theoretical framework capable of modelling all the above processes.

The R-matrix method was originally developed for nuclear physics but has proved itself outstandingly successful for the treatment of electron collisions with neutral and ionized atoms (Burke and Berrington (1993)). Indeed this method was the workhorse of the international Opacity Project which studied all atomic processes of importance for stellar atmospheres (The Opacity Project Team (1995)). Although the loss of symmetry and increased degrees of freedom mean that electron molecule collisions are significantly more difficult to treat theoretically than electron atom collisions, the use of the Rmatrix method to study low-energy electron collisions with molecules is now also well developed.

\section{The R-matrix method}

A survey of theoretical methods used to consider low-energy electron molecule collisions, including detailed consideration of the R-matrix method, can be found in Huo and Gianturco (1995). The basic idea of the R-matrix method is the division of the electron configuration space into two regions, see Fig. 1. The spherical inner region, typically of radius $10-20 \mathrm{a}_{o}$, is centered on the center of gravity of the molecule and is assumed to entirely contain the $N$ electron wavefunction of the target molecule. In this region it is necessary to consider the full, multicentered interactions between the $N+1$ electrons in the system including exchange. In the outer region it is assumed that a separate scattering electron can be identified and that exchange interactions can be neglected.

The accuracy of an R-matrix calculation is therefore crucially dependent on the representation of the problem in the inner region. In this region the scat- 

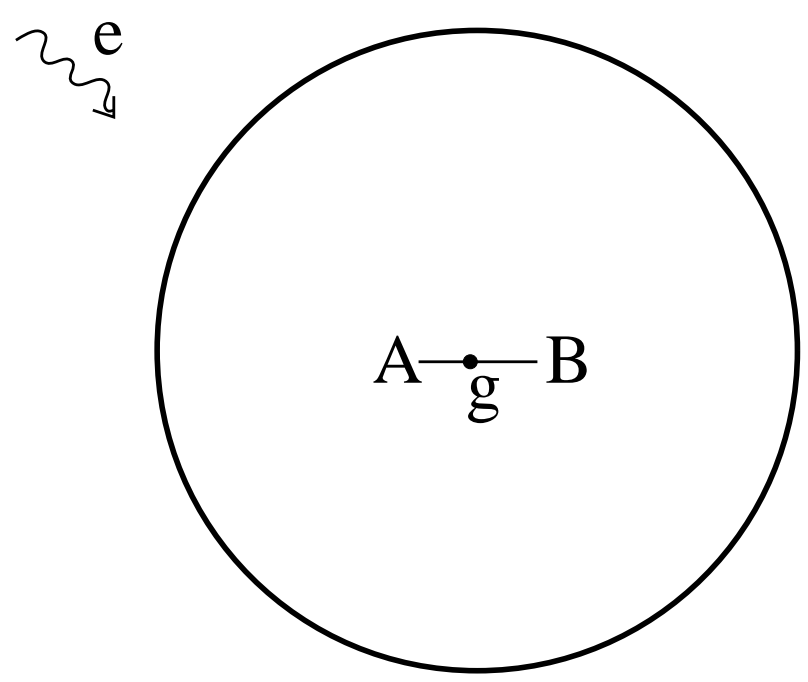

Fig. 1. R-matrix sphere for molecule AB. The sphere is centered on the center of gravity of the molecule, $\mathrm{g}$.

tering wavefunctions are written

$$
\psi_{k}=\sum_{i j} A \phi_{i}\left(x_{1} \ldots x_{N}\right) u_{i j}\left(x_{N+1}\right) a_{i j k}+\sum_{n} \chi_{n}\left(x_{1} \ldots x_{N+1}\right) b_{n k},
$$

where the sum over $i$ runs over target states and $\phi_{i}$ are $N$-electron target wavefunctions. The $u_{i j}$ are a partial wave expansion about $g$ of continuum orbitals which represent the scattering electron inside the R-matrix sphere. This electron is anti-symmetrized with respect to the target electrons by the operator $A$. The $\chi_{n}$ are two-centre quadratically integrable functions constructed from the target occupied and virtual molecular orbitals. For polyatomic molecules the target and continuum orbitals are represented by a linear combination of Gaussian Type Orbitals (GTOs), whereas for diatomics a linear combination of Slater Type Orbitals (STOs) for the target and numerical continuum functions can be used (Morgan et al. (1998)).

To allow for electron correlation, the target wavefunctions are themselves expanded as a linear combination of configurations, $\eta_{m}\left(x_{1} \ldots x_{N}\right)$, which in turn are constructed in terms of an orthonormal set of molecular orbitals,

$$
\phi_{i}=\sum_{m} \eta_{m}\left(x_{1} \ldots x_{N}\right) c_{i m}
$$

The size of this expansion is important for determining the quality of the target wavefunction.

Quantum chemists routinely perform electronic structure calculations on firstrow diatomics using many million of configurations in their calculations. Scattering calculations, even with special coding to take advantage of the structure 
of the problem (Tennyson (1996a)), are usually restricted to a few thousand configurations. Furthermore, at least in the R-matrix method, it is necessary to represent all target states included in the calculation using a single set of molecular orbitals.

The problem can be seen in terms of the R-matrix method by looking at eq (1). The number of configurations that have to be generated in a scattering calculation is the number of target configurations times the number of continuum orbitals summed over all target states. Although calculations are sometimes presented in terms of total numbers of configurations generated, this measure is somewhat method dependent. It is therefore easier and safer to make comparisons between methods in terms of target representations. The quality of target representations discussed below are similar to or better than those used by workers employing other state-of-the-art scattering methods (eg Orel et al. (1990); Rescigno (1994)).

\section{Calculations}

The UK R-matrix codes (Morgan et al. (1998)) have been used for a series of studies which treat all the processes described in the Introduction. Below we give some recent examples.

\subsection{Collisions with water}

Electron collisions with water are of particular importance for both studies of radiation damage in biological systems and atmospheric processes. We (Gorfinkiel et al. (2002)) recently completed the first study of electron impact dissociation of water to explicitly include nuclear motion, albeit only for a single, dissociative coordinate.

Figure 2 compares the electron-water molecule total cross section calculated using the lowest 7 electronic states of water in a close coupling expansion and fixing the nuclei at the equilibrium geometry, see Gorfinkiel et al. (2002) for details. Water has a large dipole moment. Experimentally this causes problems since, especially for the elastic cross section, the collisions are very strongly forward peaked making integral cross section measurements very difficult. Theoretically the large dipole means that the partial wave expansion used to represent the continuum electron is only slowly convergent. Allowance can be made for this by using the Born approximation (Chu and Dalgarno (1974)) to estimate the contribution from the higher partial waves which would otherwise be omitted. 


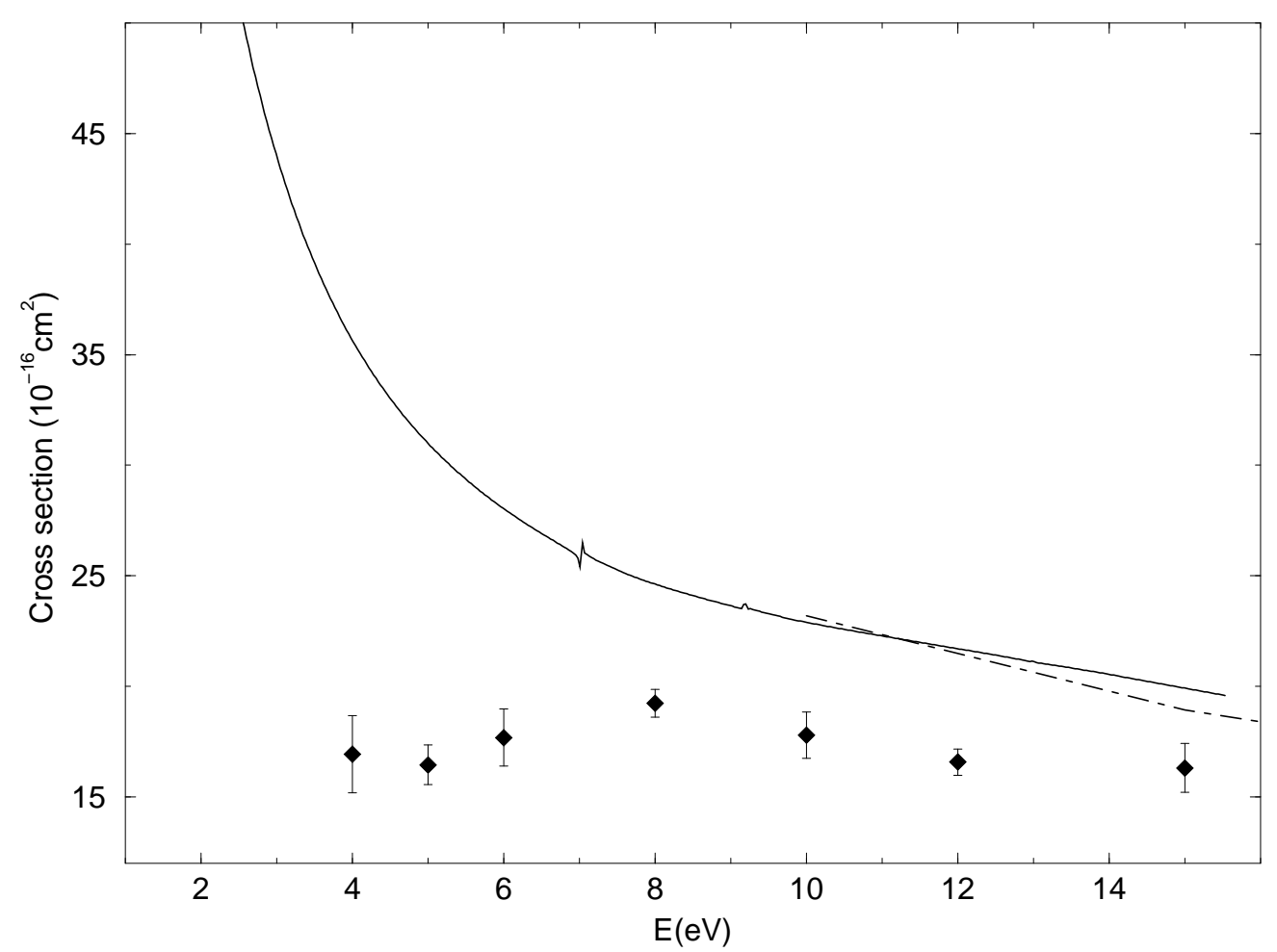

Fig. 2. Total cross section for electron collisions with water. Full line: Born corrected cross-section for a $\mathrm{J}=0$ fixed nuclei calculation Gorfinkiel et al. (2002); dotted-dashed line: Jain (1988); Experimental results by Saglam and Aktekin (1991): diamonds.

The total cross section calculated using the R-matrix method and given in figure 2 is the sum of elastic and electronically inelastic cross-sections, corrected for higher partial waves using the Born approximation. Comparison is made with the semi-empirical calculations of Jain (1988) whose model is only valid above $10 \mathrm{eV}$. At these higher energies the two calculations are in excellent agreement. Conversely, comparison with the total cross section measurements of Saglam and Aktekin (1991) show a significant discrepency. This discrepency is not unexpected. The total cross section is dominated by the elastic cross section and previous comparisons, see Gorfinkiel et al. (2002), show that theory consistently gives higher elastic cross sections than experiment. Since it is possible to obtain good agreement with the elastic angular differential cross sections at intermediate angles, it would appear that this discrepency is due to the difficulty of obtaining reliable experimental measurements for forward scattering.

\subsection{Electron collisions with the CF radical}

The etching of silicon wafers using molecular plasmas is the major technology driving the manufacture of small computer components. However the processes 

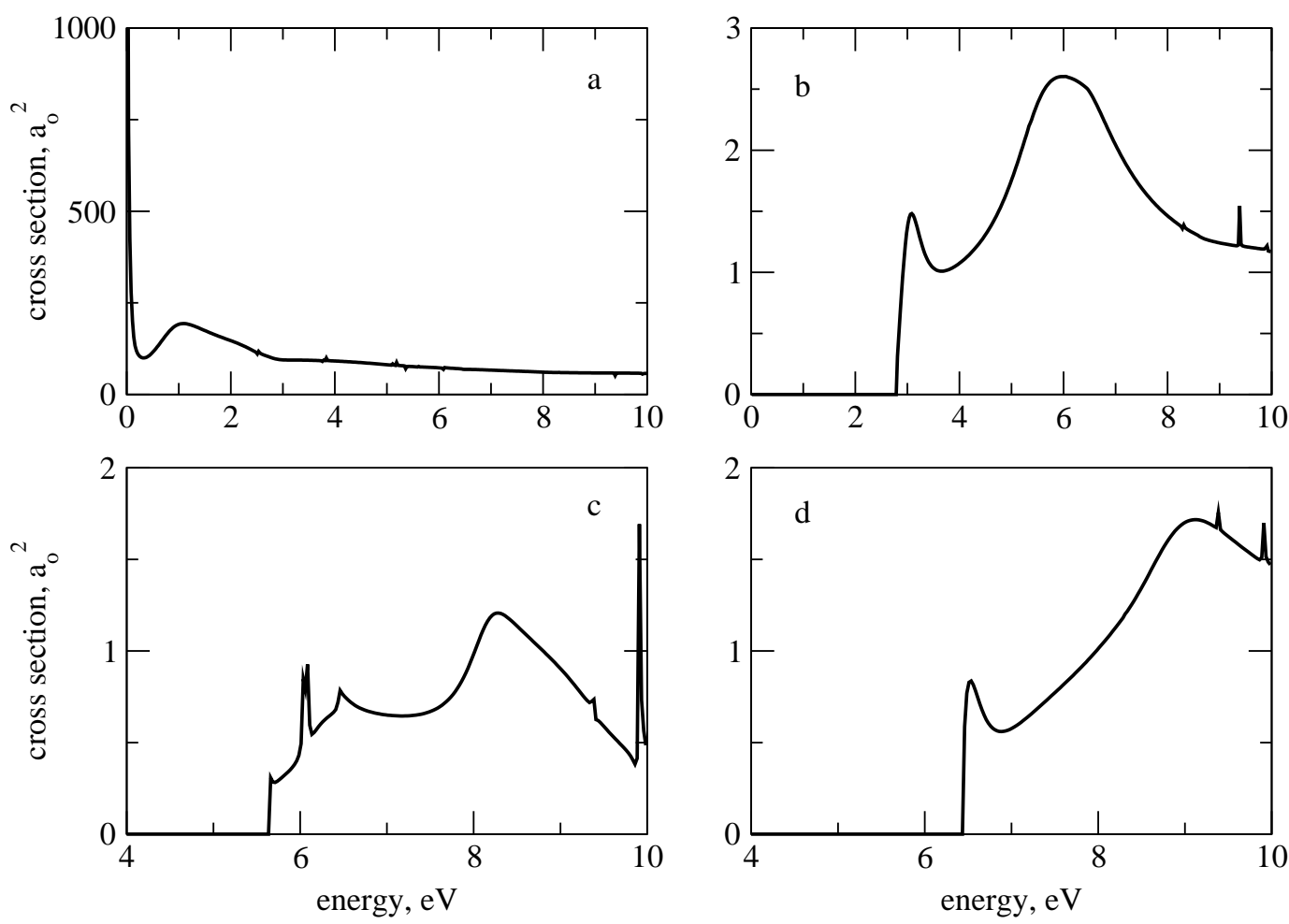

Fig. 3. Calculated cross sections for electron impacts on $\mathrm{CF}$ in its $\mathrm{X}{ }^{2} \Pi$ ground state and equilibrium geometry.a elastic collisions; b excitation to the a ${ }^{4} \Sigma^{-}$state; c excitation to the $\mathrm{A}^{2} \Sigma^{+}$state; d excitation to the $\mathrm{B}^{2} \Delta$ state.

which occur in etching plasmas are poorly characterized, in part because of their variety and complexity. We are performing a series of calculation on the $\mathrm{CF}_{x}(x=1,2,3)$ radicals; these species arise from fragmentation of the $\mathrm{CF}_{3} \mathrm{I}$ and $\mathrm{C}_{2} \mathrm{~F}_{4}$ molecules which are presently being investigated as possible feedstock gases for plasma etching. Electron collisions with stable species such as $\mathrm{CF}_{3} \mathrm{I}$ are amenable to experimental investigation (Mason et al. (2003)). However, experimental studies on highly reactive radicals are extremely difficult. $A b$ initio calculations can therefore play an important role in providing data upon which models can be based (Winstead and McKoy (2000)). The $\mathrm{CF}_{x}$ species are electron rich making them particularly demanding theoretically. Results for electron collisions with $\mathrm{CF}_{2}$ have already been reported (Rozum et al. (2002)).

Figure 3 presents preliminary results for electron collisions with the CF radical frozen at its equilibrium geometry. Like water, $\mathrm{CF}$ also has a large dipole and to obtain reliable elastic and dipole-allowed electronic cross selections it will be necessary to top up our R-matrix calculations using the Born approximation. The particularly large value for the elastic cross section at low energy, see Figure 3a will undoubtedly be reduced by the proper treatment of rotational motion, see Baluja et al. (2001). 


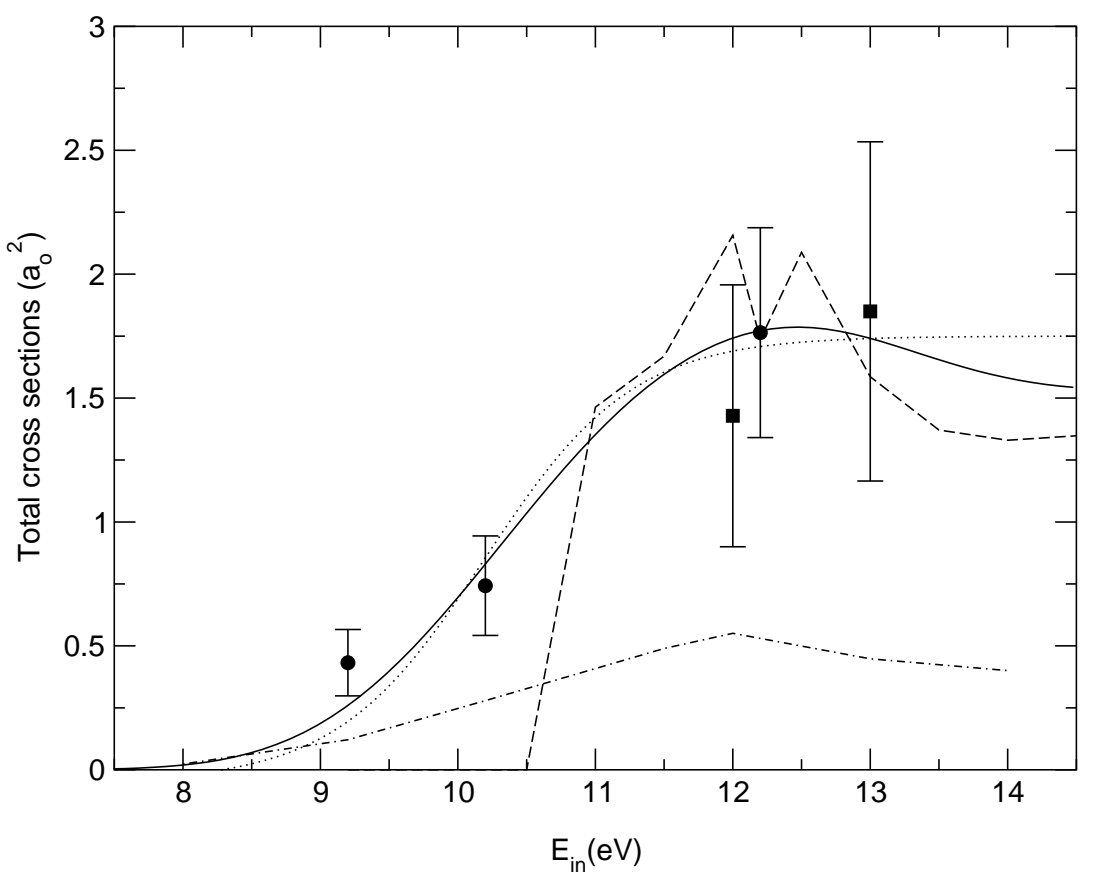

Fig. 4. Electron impact dissociation cross sections for $\mathrm{H}_{2}(v=0)$. Theoretical results are given by: solid curve: full adiabatic nuclei calculation (Trevisan and Tennyson (2001)); dotted curve: scaling law fit (Trevisan and Tennyson (2002b)); dashed curve: fixed nuclei approximation: chain curve: delta-function approximation. Experimental results are from Khakoo and Segura (1994): circles and Nishimura and Danjo (1986): squares.

The cross sections depicted in figure 3 display a number of features which can be ascribed to resonances. These resonances are of particular importance for the plasma etching species since they provide a chemically important route to dissociation via dissociative attachment. Calculations giving the behaviour of the resonances as a function of CF bondlength will be reported elsewhere (Rozum et al. (2003)).

\subsection{Electron impact dissociation of molecular hydrogen}

Electron molecule collisions are also important at the edge of fusion plasmas. We have recently completed a series of studies on the near-threshold electron impact dissociation of $\mathrm{H}_{2}$ and its isotopomers (Trevisan and Tennyson (2001, $2002 \mathrm{a}, \mathrm{b})$ ). In the near threshold region nuclear motion effects are particularly important, with the rate of electron impact dissociation showing a strong dependence on the initial vibrational state of the molecular hydrogen and on the reduced mass of the isotopomer.

Figure 4 compares impact dissociation cross sections for $\mathrm{H}_{2}$ in its vibrational ground state. It can be seen that the fixed nuclei approximation is poor in 
the near threshold region and in particular does not give the correct effective threshold. This approximation becomes increasingly reliable at higher impact energies (not shown). Note that the structure in the fixed-nuclei cross sections is caused by resonances which are not fully resolved by the energy grid used for the calculations. In contrast to the fixed nuclei calculations, the delta function approximation appears to only give reliable results very close to the effective threshold for the excitation process.

One issue of interest for models of fusion plasmas is the kinetic energy of the atoms which result from the impact dissociation process. A full theory for the such energy (and angular) differential cross sections has only just been formulated (Trevisan and Tennyson (2001)). Figure 5 gives energy differential cross sections for impact dissociation of HT as function of the initial vibrational state of the molecule, $v$. It can be seen that the energy of the outgoing atomic fragment depends quite significantly on $v$ with the peak fragment energy generally dropping with $v$.

\subsection{Dissociative recombination of $\mathrm{CO}^{2+}$}

The completely $a b$ initio calculation of dissociative recombination cross sections or rates is generally very demanding because of the high sensitivity of this process to the location of the exact crossing point between ion and resonance state energy curves (Tennyson (1996b)). However with the correct curve crossings it is possible to get very good agreement with the high quality experimental data now available from storage rings, see Schneider et al. (2000) for example.

Recent experiments using the Astrid storage ring have studied dissociative recombination of doubly charged molecular ions for the first time. Of particular interest are the experiments on $\mathrm{CO}^{2+}$ (Safvan et al. (1999)) given the astronomical abundance of $\mathrm{CO}$.

We are presently studying electron collisions with $\mathrm{CO}^{2+}$. As a first stage in any such calculation it is necessary to characterize the low-lying target states of the system. In the case of $\mathrm{CO}^{2+}$ it transpires that there are a number of very low-lying electronic states. Figure 6 shows our calculations for the lowest 7 electronic states of $\mathrm{CO}^{2+}$; these curves are in good agreement with the previous calculations of Larsson et al. (1989) and Andersen et al. (1993), and are consistent with the near-threshold photo-double ionisation experiments on CO performed by Dawber et al. (1994).

The small gap between the $\mathrm{CO}^{2+}$ ground state curve and the excited states means that large numbers of resonance states will need to be considered. The inset illustrates this for the case of a $\mathrm{CO}^{+}$Rydberg state with principle 

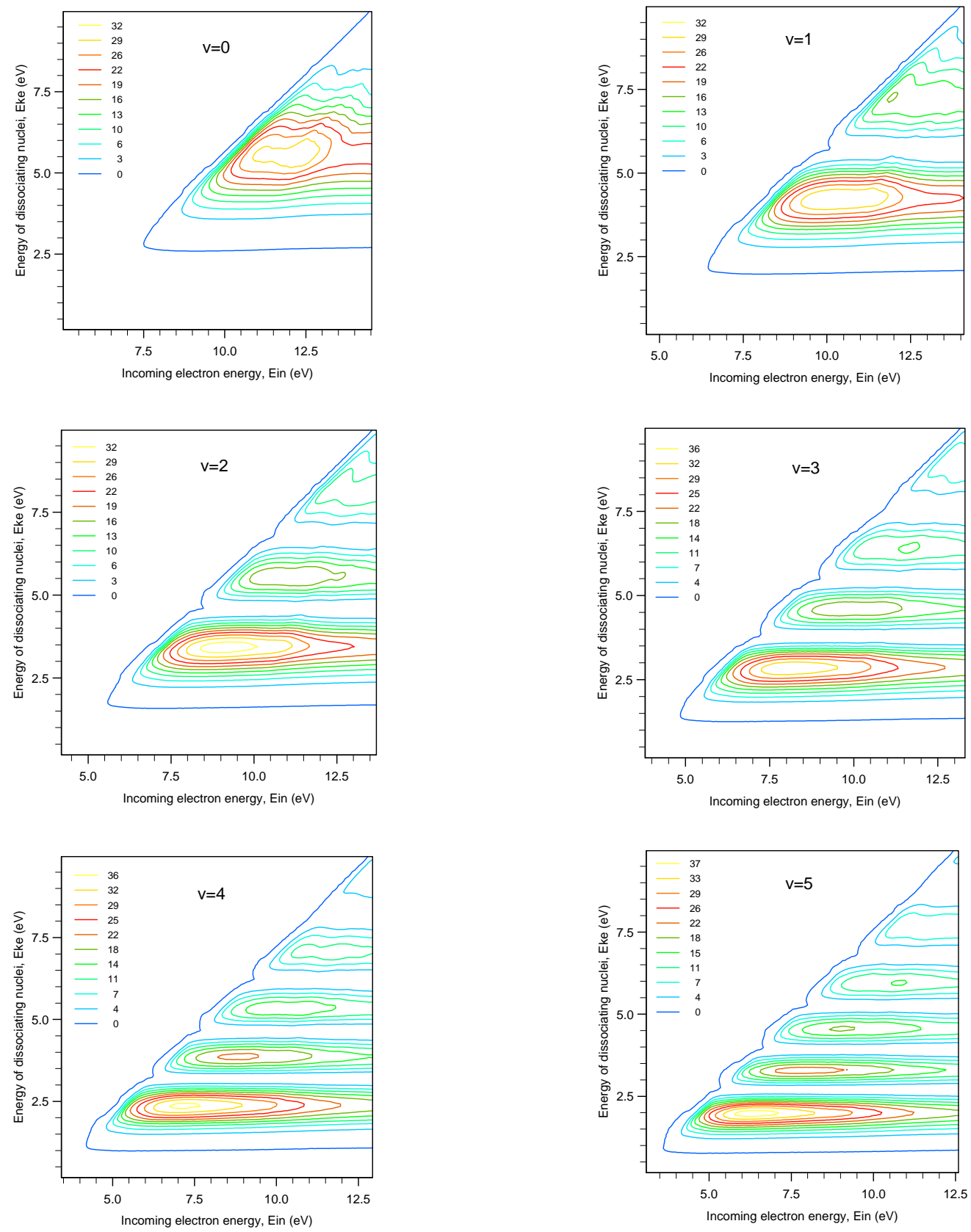

Fig. 5. Energy differential cross sections, in atomic units, for electron impact dissociation of $\operatorname{HT}(v)$, as a function of initial vibrational state, $v$. The energy of the dissociating atoms, $E_{k e}$, is plotted against the different values of incoming electron energies, $E_{i n}$. The structure of the bound vibrational wavefunction can be seen as $v$ increases from $v=0$ to $v=5$.

quantum $n=20$ associated with each of the three lowest target states. As for diatomics there are approximately $n$ Rydberg states with principle quantum number $n$, it is clear that any detailed study will need to consider large number of $\mathrm{CO}^{+}$resonances. This should be achievable within the context of 


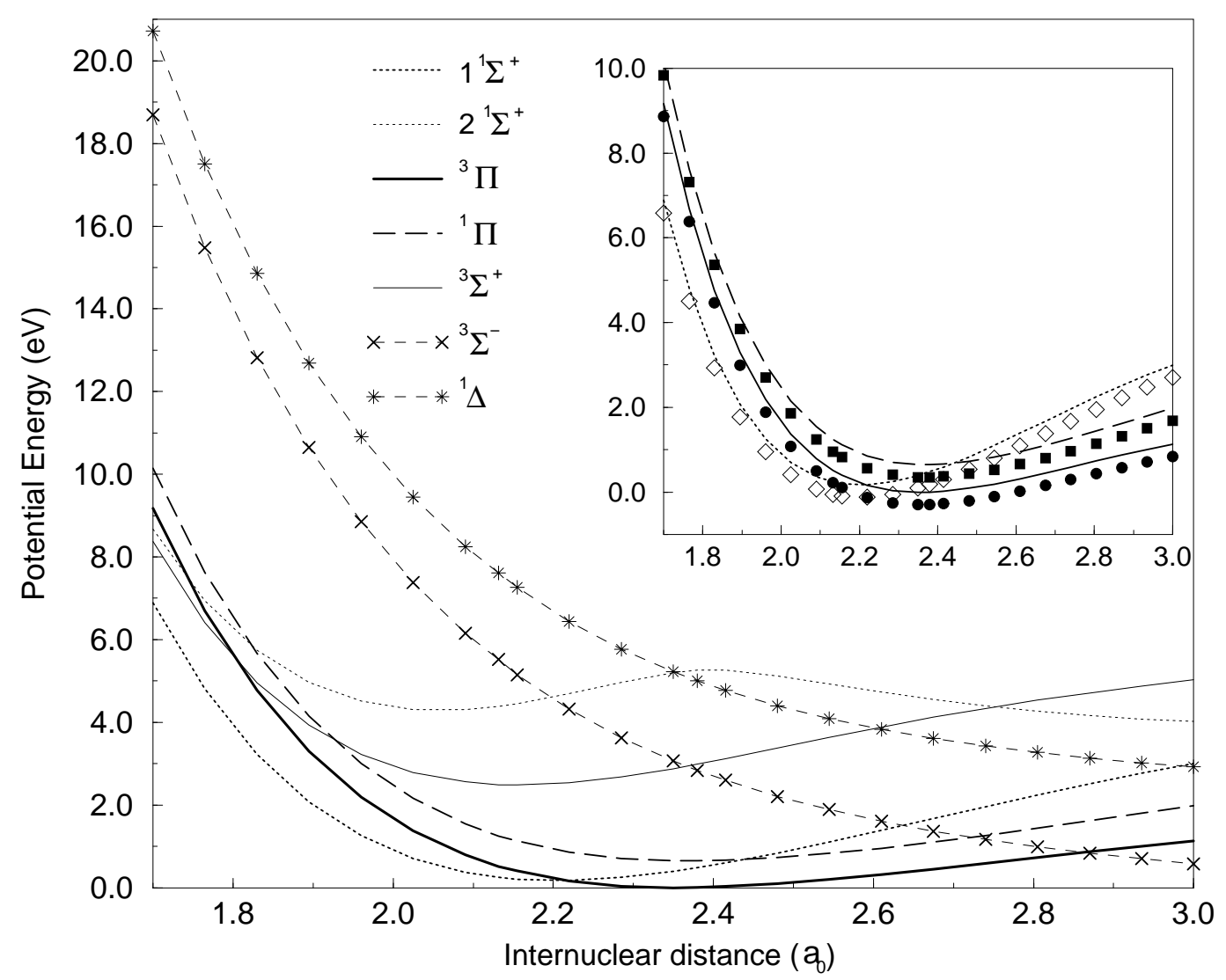

Fig. 6. Low-lying potential energy curves for $\mathrm{CO}^{2+}$. The inset shows the lowest three curves and, for each curve, an associated high-lying Rydberg state of $\mathrm{CO}^{+}$ with principal quantum number $n=20$.

multichannel quantum defect theory (Jungen (1995)).

\section{Conclusions}

The examples discussed here are not a comprehensive list of recent calculations. In particular neither electron impact rotational nor vibrational excitation calculations are considered above. In fact recent R-matrix calculations have studied electron impact rotational excitation of molecular ions in some detail, with a comprehensive study of astronomically important diatomic ions (Faure and Tennyson (2001)) which has recently been extended to the problem of symmetric tops (Faure and Tennyson (2002)). At present we are working on further extensions of the method to include, for example, multidimensional nuclear motion for polyatomic systems and extending the energy range treated to cover the so-called intermediate energy region which spans the first ionisation threshold of the target. 


\section{Acknowledgements}

We thank Nigel Mason for helpful discussions and Lesley Morgan for help in developing and maintaining the codes. This work has been supported by the EPSRC under a variety of grants and the European Union as part of the ETR and EPIC networks.

\section{References}

Andersen, L. H., Posthumus, J. H., Vahtras, O., Ågren, H., Elander, N., Nunez, A., Scrinzi, A., Natiello, M., Larsson, M., 1993. Very slow spontaneous dissociation of $\mathrm{CO}^{2+}$ observed by means of a heavy-ion storage-ring. Phys. Rev. Lett. 71, 1812-1815.

Baluja, K. L., Mason, N. J., Morgan, L. A., Tennyson, J., 2001. Electron collisions with $\mathrm{OClO}$ using the R-matrix method. J. Phys. B: At. Mol. Opt. Phys. 34, 4041-4052.

Burke, P. G., Berrington, K. A. (Eds.), 1993. Atomic and Molecular Processes, an R-matrix Approach. Institute of Physics Publishing, Bristol.

Chu, S.-I., Dalgarno, A., 1974. Rotational excitation of $\mathrm{CH}^{+}$by electron impact. Phys. Rev. A 10, 788-92.

Dawber, G., McConkey, A. G., Avaldi, L., MacDonald, M. A., King, G. C., Hall, R. I., 1994. Theshold photoelectrons coincidence spectroscopy of doubly-charged ions on nitrogen, carbon-monoxide, nitric-oxide and oxygen. J. Phys. B: At. Mol. Opt. Phys. 27, 2191-2209.

Faure, A., Tennyson, J., 2001. Electron-impact rotational excitation of linear molecular ions. Mon. Not. R. astr. Soc. 325, 443-448.

Faure, A., Tennyson, J., 2002. Electron-impact rotational excitation of symmetric top molecular ions. J. Phys. B: At. Mol. Opt. Phys. 35, 3945-3956.

Gorfinkiel, J. D., Morgan, L. A., Tennyson, J., 2002. Electron impact dissociative excitation of water within the adiabatic nuclei approximation. J. Phys. B: At. Mol. Opt. Phys. 35, 543-555.

Huo, W. M., Gianturco, F. A. (Eds.), 1995. Computational Methods for Electron Molecule Collisions. Plenum Press, New York, 233 Spring Street, New York, NY 10013, USA.

Jain, A., 1988. Theoretical study of the total (elastic+inelastic) cross sections for electron $-\mathrm{H}_{2} \mathrm{O}\left(\mathrm{NH}_{3}\right)$ scattering at 10-3000 eV. J. Phys. B: At. Mol. Opt. Phys. 21, 905-924.

Jungen, C. (Ed.), 1995. Molecular Applications of Quantum Defect Theory. IOP Press, Bristol.

Khakoo, M. A., Segura, J., 1994. Differential cross sections for the electron impact excitation of the $\mathrm{B}^{3} \Sigma_{u}^{+}$continuum of molecular hydrogen. J. Phys. B: At. Mol. Opt. Phys. 27, 2355-2368. 
Larsson, M., Olsson, B. J., Sigray, P., 1989. Theoretical study of the $\mathrm{CO}^{2+}$ dication. Chem. Phys. 139, 457-469.

Mason, N. J., Limao Viera, P., Eden, S., Kendall, P., Pathak, S., Dawes, A., Tennyson, J., Tegeder, P., Kitajima, M., 2003. VUV and low energy electron impact study of electronic state spectroscopy of $\mathrm{CF}_{3} \mathrm{I}$. J. Phys. B: At. Mol. Opt. Phys. .

Morgan, L. A., Tennyson, J., Gillan, C. J., 1998. The UK molecular R-matrix codes. Computer Phys. Comms. 114, 120-128.

Nishimura, H., Danjo, A., 1986. Differential cross-section of electron-scattering molecular-hydrogen 2. B ${ }^{3} \Sigma_{u}^{+}$excitation. J. Phys. Soc. Japan 55, 3031-3036.

Orel, A. E., Rescigno, T. N., Lengsfield III, B. H., 1990. Theoretical study of electron-impact excitation of $\mathrm{N}_{2}^{+}$. Phys. Rev. A 42, 5292.

Rescigno, T. N., 1994. Low-energy electron-collision processes in molecular chlorine. Phys. Rev. A 50, 1382-1389.

Rozum, I., Mason, N. J., Tennyson, J., 2002. Electron collisions with the $\mathrm{CF}_{2}$ radical using the R-matrix method. J. Phys. B: At. Mol. Opt. Phys. 35, $1583-1591$.

Rozum, I., Mason, N. J., Tennyson, J., 2003. Electron collisions with CF using the R-matrix method. J. Phys. B .

Safvan, C. P., Jensen, M. J., Pendersen, H. B., Andersen, L. H., 1999. Dissociative recombination of the $\mathrm{CO}^{2+}$ dication. Phys. Rev. A 60, R3361-R3364.

Saglam, Z., Aktekin, N., 1991. Absolute total cross sections for scattering of electrons by $\mathrm{H}_{2} \mathrm{O}$ in the energy range $4-20 \mathrm{eV}$. J. Phys. B: At. Mol. Opt. Phys. 24, 3491.

Schneider, I. F., Rabadán , I., Carata, L., Tennyson, J., Andersen, L. H., Suzor-Weiner, A., 2000. Dissociative recombination of $\mathrm{NO}^{+}$: theoretical modelling and comparison with measurements. J. Phys. B: At. Mol. Opt. Phys. 33, 4849-4861.

Tennyson, J., 1996a. A new algorithm for hamiltonian matrix construction in electron-molecule collision calculations. J. Phys. B: At. Mol. Opt. Phys. 29, $1817-1828$.

Tennyson, J., 1996b. Theory of low energy electron-molecule dissociative recombination. Comments At. Mol. Phys. 32, 209-218.

The Opacity Project Team, 1995. The Opacity Project Volume 1. IOP Press, Bristol.

Trevisan, C. S., Tennyson, J., 2001. Differential cross sections for near threshold electron impact dissociation of molecular hydrogen. J. Phys. B: At. Mol. Opt. Phys. 34, 2935-2949.

Trevisan, C. S., Tennyson, J., 2002a. Calculated rates for the electron impact dissociation of molecular hydrogen, deuterium and tritium. Plasma Phys. Controlled Fusion 44, 1263-1276.

Trevisan, C. S., Tennyson, J., 2002b. Calculated rates for the electron impact dissociation of molecular hydrogen: mixed isotopomers and scaling laws. Plasma Phys. Controlled Fusion 44, 2217-2230.

Winstead, C., McKoy, V., 2000. Electron-molecule collisions in low- 
temperature plasmas - the role of theory. Adv. Atom. Mol. Opt. Phys. 43, 111-145. 